\title{
A Full-Wave Mixed Potential Mode-Matching Method for the Analysis of Planar or Quasi-Planar Transmission Lines
}

\author{
Ching-Kuang C. Tzuang, Member, IEEE, and Jan-Dong Tseng, Student Member, IEEE
}

\begin{abstract}
A newly proposed and tested full-wave mixed potential mode-matching method is presented for the analysis of planar and/or quasi-planar transmission lines. The transmission lines under investigation consist of layered (stratified) and nonlayered dielectric substrates and metal strips of finite thickness. The $y$-directed hybrid TE and TM Hertzian potentials, perpendicular to the interfaces between each layered region, are employed in the layered regions. The nonlayered regions, which consist of dielectric step discontinuities that destroy the layered configuration in the horizontal plane, utilize the $x$-directed hybrid TE and TM Hertzian potentials parallel to the horizontal plane. As a direct result, the full-wave formulation of the transmission line problem becomes systematic and easy to handle. Extensive analyses of a particular case study show that the relative convergence criterion needs to be satisfied to obtain accurate electromagnetic field solutions. The theoretical results obtained here are in very good agreement with the published data for various transmission line structures, which are the special limiting cases of the particular case study. The applications of the new formulation to the proximity effects of microstrip and Microslab ${ }^{1}$ lines are also illustrated by examples.
\end{abstract}

\section{INTRODUCTION}

$\mathrm{M}$ ONOLITHIC microwave integrated circuits (MMIC's) and lightwave technologies have proliferated and demonstrated the potential for extensive use in military and civilian communication systems [1], [2]. The capability of achieving first-pass success in MMIC chip design will improve the competitiveness of GaAs MMIC vendors [3] and is closely related to the so-called producibility engineering of MMIC's [1], [3]. The key to the first-time design success is the utilization of accurate simulation tools at the subsystem, circuit, device, and process levels in an integrated CAD-based environment [1].

Motivated by the need for providing accurate, realistic, and reliable models for the planar and quasi-planar transmission lines in addition to the existing MMIC CAD

Manuscript received November 26, 1990: revised May 23, 1991. This work was supported by the National Science Council, Republic of China, under Grant NSC80-0404-E009-50 and Contract CS80-(1210D009-21.

The authors are with the Institute of Communication Engineering and the Microelectronics and Information Science and Technology Research Center, National Chiao Tung University, No. 75, Po-Ai Street, Hsinchu Taiwan, Republic of China.

IEEE Log Number 9102336.

${ }^{1}$ Microslab is a trademark of the Martin Marietta Corporation.

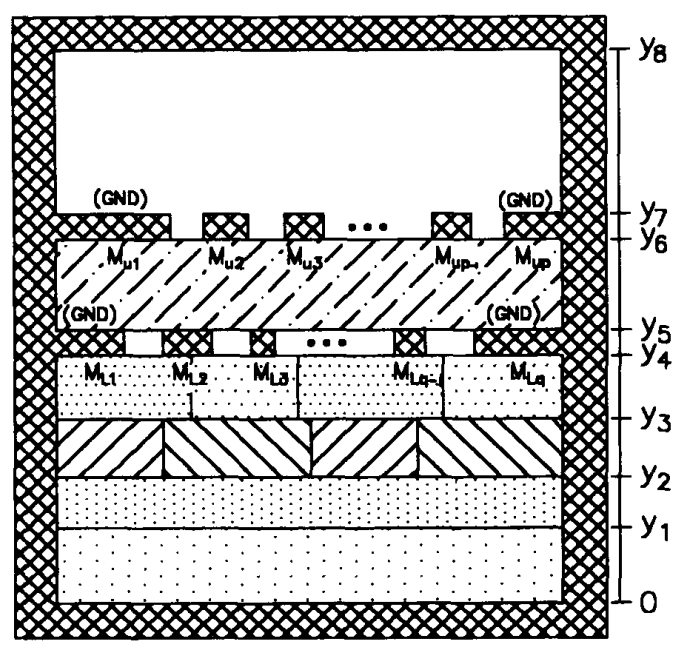

Fig. 1. A generalized planar and/or quasi-planar transmission lines structure for microwave, millimeter-wave, and optoelectronic applications.

software, this paper proposes a full-wave field-theorybased technique to solve the propagation characteristics of the quasi-planar transmission lines shown in Fig. 1, which consists of finite-thickness coupled metal strips embedded in multidielectric media. These dielectric substrates are of two types. One is commonly known as the layered (or stratified) configuration, defined in regions $y \in\left(0, y_{1}\right),\left(y_{1}, y_{2}\right),\left(y_{5}, y_{6}\right)$, and $\left(y_{7}, y_{8}\right)$. The other is the nonlayered configuration, defined in regions $y \in\left(y_{2}, y_{3}\right)$ and $\left(y_{3}, y_{4}\right)$. In the nonlayered configuration, obviously, dielectric discontinuities in the horizontal $(x)$ direction occur. Because of the existence of the nonlayered configuration, a full-wave formulation of the transmission line becomes difficult. The metal strips have finite thickness, which has a noticeable effect on tightly coupled microstrip lines and finline structures [3]-[5]. Note that the metal strips are assumed to be perfect, without ohmic losses.

The application of the transmission line model shown in Fig. 1 or its simplified configuration shown in Fig. 2(a) lies in most lightwave or millimeter-wave circuits. The optoelectronic integrated circuits (OEIC's) that monolith- 


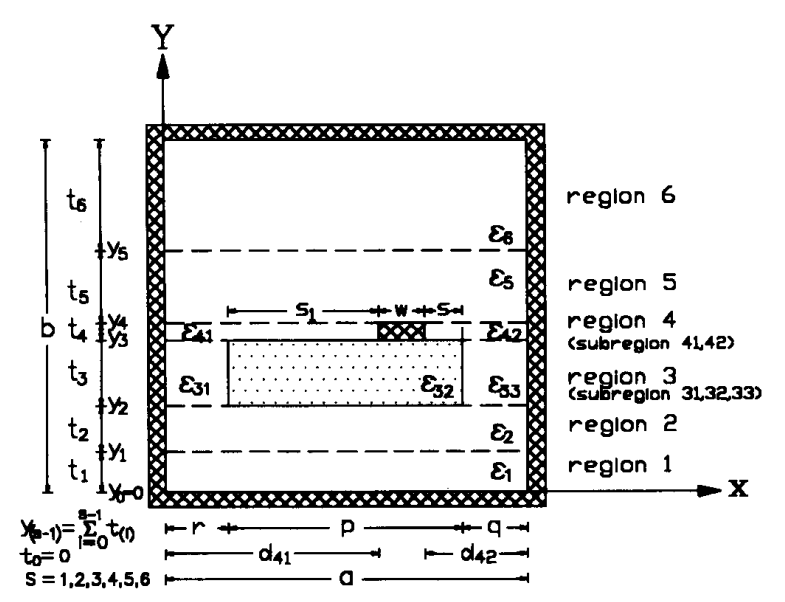

(a)

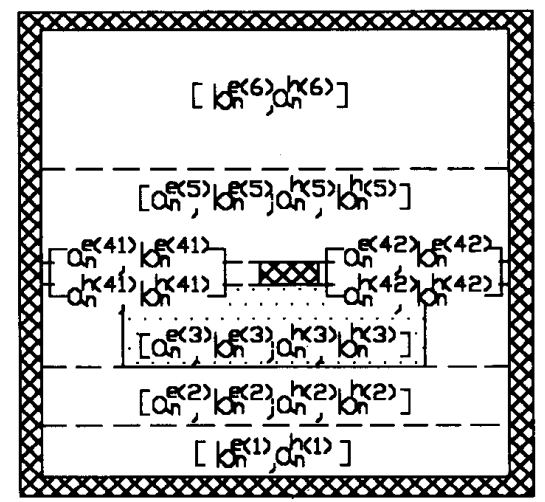

(b)

Fig. 2. A cross section of a transmission line of finite thickness integrated on layered (stratified) and nonlayered substrates. (a) Designation of the corresponding structural and material parameters used throughout this paper. (b) The associated unknown coefficients in each region for use in the proposed mixed potential mode-matching formulation described in Section II. Superscripts $e$ and $h$ denote TM and TE modes, respectively.

ically integrated both optical and electrical components on the same chip often mandate nonlayered guided-wave structures. For instance, when integrating MSM (metal-semiconductor-metal) photodectors with FET amplifiers in OEIC's, the metal strips for the MSM photodectors and FET's are in different layers with dielectric step discontinuities between the adjacent MSM photodector and FET [6, fig. 19]. Another application is the rigorous study of the propagation characteristics of travelingwave electro-optic modulators, which may consist of ridge or strip-loaded structures [7], [8] or certain nonlayered dielectric substrates [9, fig. 1(a)].

Figs. 1 and 2(a) also have many interesting applications in millimeter-wave circuits. When fabricating a W-band monolithic cross bar mixer, an epitaxial layer structure of $\mathrm{n}-\mathrm{n}^{+}$grown in a semi-insulating substrate followed by proton bombardment for isolation will result in a nonlayered region as shown in Fig. 1 [10, fig. 4]. In the second example, Young and Itoh proposed a novel superconduct- ing ridged microstrip to reduce dielectric and conductor losses [11]. Both ridged microstrip and Microslab [12] structures are special cases of Figs. 1 and 2(a). In the third example, a technique for improving the MMIC slotline resonator by reactive ion etching of the GaAs substrate also results in a nonlayered region [13, fig.. 1]. Finally, the proximity effects pointed out by Pucel [14] can also be easily described by Fig. 1. Pucel defined two types of proximity effects. The first is a microstrip line near the edge of an MMIC substrate. The second is a microstrip line adjacent to a coplanar ground plane. Moreover, using the full-wave approach, the tangled effect of the nearby housing and the proximity effects can be analyzed accurately based on our formulation approach.

Thus it is very important to develop a full-wave fieldtheory-based CAD program to model various transmission lines containing nonlayered structures for use in MMIC's and optoelectronic circuits. The techniques available for solving the guided-wave problems include the finite difference method (FDM) [15], the finite element method (FEM) [16], [17], the boundary integral equation method (BIEM) [18], the point-matching method [19], the method of lines [20], the mode-matching method [11], [12], the finite difference time domain method [21], and the transmission line matrix method [22]. Methods such as the FDM, FEM, BIEM, and point-matching method are in general subject to spurious solutions when used in the full-wave analysis of guided-wave structures with sharp metal edges [18], and will entail extra time and effort in discriminating spurious and physical solutions. This paper, however, presents a new mode-matching formulation technique using the Hertzian potentials in both the transverse $x$ (horizontal) and $y$ (vertical) directions of Fig. 2(a) and extends the new method to such an extent that it is as flexible as the method of lines [20] for analyzing planar and quasi-planar transmission lines containing nonlayered substrates.

The new formulation is based on the fact that the Hertzian potentials can be expanded in arbitrary directions when constructing the full-wave solutions [23]. When closely observing the transmission line models shown in Fig. 1 or Fig. 2(a), the dielectric discontinuities occur in the $y$ direction for the layered regions whereas the dielectric step discontinuities appear in the $x$ direction for the nonlayered regions. Consequently it becomes a natural choice to use the $y$-directed and $x$-directed hybrid TE and TM Hertzian potentials for the layered and nonlayered regions, respectively. This in turn matches the boundary conditions inside the layered and nonlayered regions. The efforts necessary to formulate the proposed mixed potential mode-matching method is then greatly reduced because few boundary conditions are left to be met. Note that in the conventional [12], [24] and modified [25], [26] mode-matching methods, the waveguides under analysis consist of layered substrates and the Hertzian potentials employed are in one direction only.

Section II describes in detail the proposed mixed potential mode-matching formulation. The formulation, 
however, can be extended to analyze the waveguide structure shown in Fig. 1 without much difficulty. Section III performs the convergence study of the proposed method. The rigorous spurious-mode-free solutions for the waveguide structure shown in Fig. 2(a) are obtained and reported. The proximity effects of a shielded microstrip and a modified Microslab are presented in Sections IV and V, respectively. Their physical implications are also discussed. Finally Section VI concludes this paper.

\section{Formulation: Mixed Potential Mode-Matching Method}

In this section we will use the simplified configuration of Fig. 1, namely, Fig. 2(a), as a test case to illustrate the procedure of formulating the proposed mixed potential mode-matching method. Fig. 2(a) is segmented into various regions. Regions $1,2,5$, and 6 belong to the layered (stratified) regions. On the other hand region 3 is subdivided into subregions 31,32 , and 33 , which constitute the nonlayered region. Region 4 consists of a perfectly conducting metal strip of finite thickness and the subregions 41 and 42 next to the metal strip. The entire guided-wave structure is enclosed by a perfectly conducting rectangular housing.

Assuming a time-harmonic factor $e^{j \omega t}$, in a homogeneous source-free region, the electromagnetic fields in terms of Hertzian potentials satisfying the Helmholtz equation are

$$
\begin{aligned}
\overline{\boldsymbol{E}}^{(s)} & =\boldsymbol{\nabla} \times \boldsymbol{\nabla} \times \overline{\boldsymbol{\pi}}_{e}^{(s)}-j \omega \mu \boldsymbol{\nabla} \times \overline{\boldsymbol{\pi}}_{h}^{(s)} \\
\overline{\boldsymbol{H}}^{(s)} & =j \omega \epsilon \boldsymbol{\nabla} \times \overline{\boldsymbol{\pi}}_{e}^{(s)}+\boldsymbol{\nabla} \times \boldsymbol{\nabla} \times \overline{\boldsymbol{\pi}}_{h}^{(s)} .
\end{aligned}
$$

Since the solutions for the Helmholtz equation are separable in Cartesian coordinates, $\bar{\pi}_{e}^{(s)}(x, y, z)$ and $\bar{\pi}_{h}^{(s)}(x, y, z)$ in (1a) and (1b) can be expressed as

$$
\begin{aligned}
& \overline{\boldsymbol{\pi}}_{e}^{(s)}(x, y, z)=\sum_{n=p}^{N_{e}^{(s)}} \phi_{n}^{e(s)}(x) \psi_{n}^{e(s)}(y) e^{-j \gamma z} \overline{\boldsymbol{e}}^{(s)} \\
& \overline{\boldsymbol{\pi}}_{h}^{(s)}(x, y, z)=\sum_{n=q}^{N_{h}^{(s)}} \phi_{n}^{h(s)}(x) \psi_{n}^{h(s)}(y) e^{-j \gamma z} \overline{\boldsymbol{e}}^{(s)}
\end{aligned}
$$

where $s$ denotes the index of a region or a subregion corresponding to Fig. 2(a). $\overline{\boldsymbol{e}}^{(s)}$ is a unity constant vector in the $x$ direction when $s$ is equal to $31,32,33,41$, and 42 and in the $y$ direction when $s$ is equal to $1,2,5$, and 6 in the formulation. The value of $p(q)$ is either 0 or 1 when $\bar{e}^{(s)}$ is in the $x(y)$ or $y(x)$ direction respectively. In what follows, subsections II-A and II-B describe the setup of the Hertzian potentials for the layered and nonlayered regions and the slot regions. Subsection II-C lists all the boundary conditions in the horizontal plane. Subsection II-D explains how to incorporate the boundary conditions and derives the final nonstandard eigenvalue equation by eliminating the coefficients associated with the Hertzian potentials in each region.

\section{A. Eigenfunction Expansions for the Layered (Stratified)} Regions ( $s=1,2,5$, and 6 )

Referring to Fig. 2(a), the hybrid TE-to- $y$ and TM-to-y Hertzian potentials of $(2 a)$ and $(2 b)$ in the layered regions, where $s=1,2,5$, and 6 , can be expressed as follows:

$$
\begin{aligned}
\phi_{n}^{e(s)}(x)= & \sin [(n \pi / a) x] \\
\phi_{n}^{h(s)}(x)= & \cos [(n \pi / a) x] \\
\psi_{n}^{e(s)}(y)= & a_{n}^{e(s)} \cos \left[\beta_{y n}^{(s)}\left(y-y_{(s-1)}\right)\right] \\
& +b_{n}^{e(s)} \sin \left[\beta_{y n}^{(s)}\left(y-y_{(s-1)}\right)\right] \\
\psi_{n}^{h(s)}(y)= & a_{n}^{h(s)} \cos \left[\beta_{y n}^{(s)}\left(y-y_{(s-1)}\right)\right] \\
& +b_{n}^{h(s)} \sin \left[\beta_{y n}^{(s)}\left(y-y_{(s-1)}\right)\right] \\
\psi_{n}^{e(s)}(y)= & a_{n}^{e(s)} \cos \left[\beta_{y n}^{(s)}\left(y_{(s)}-y\right)\right] \\
& +b_{n}^{e(s)} \sin \left[\beta_{y n}^{(s)}\left(y_{(s)}-y\right)\right] \\
\psi_{n}^{h(s)}(y)= & a_{n}^{h(s)} \cos \left[\beta_{y n}^{(s)}\left(y_{(s)}-y\right)\right] \\
& +b_{n}^{b(s)} \sin \left[\beta_{y n}^{(s)}\left(y_{(s)}-y\right)\right]
\end{aligned}
$$

where

$$
\begin{array}{cc}
y_{(s-1)}=\sum_{i=1}^{s-1} t_{(i)} & t_{0}=0 \\
\left(\beta_{y n}^{(s)}\right)^{2}=\epsilon_{r}^{(s)} k_{0}^{2}-(n \pi / a)^{2}-\gamma^{2}, & \left(k_{0}^{2}=\omega^{2} \mu_{0} \epsilon_{0}\right) .
\end{array}
$$

Expressions (4a) and (4b) are applied in regions 1 and 2, and expressions (4c) and (4d) are applied in regions 5 and 6 , respectively.

\section{B. Eigenfunction Expansions for the Nonlayered Regions} $(s=31,32,33)$ and the Slot Regions $(s=41,42)$ Next to the Metal Strips

In the nonlayered regions of Fig. 2(a), where $s=31,32$, and 33, the hybrid TE-to- $x$ and TM-to- $x$ Hertzian potentials of $(2 a)$ and $(2 b)$ are

$$
\begin{aligned}
\phi_{n}^{e(s)}(x)= & c_{n}^{e(s)} \cos \left[\beta_{x n}^{e(s)}\left(x-x_{(s)}\right)\right] \\
& +d_{n}^{e(s)} \sin \left[\beta_{x n}^{e(s)}\left(x-x_{(s)}\right)\right] \\
\phi_{n}^{h(s)}(x)= & c_{n}^{h(s)} \cos \left[\beta_{x n}^{h(s)}\left(x-x_{(s)}\right)\right] \\
& +d_{n}^{h(s)} \sin \left[\beta_{x n}^{h(s)}\left(x-x_{(s)}\right)\right]
\end{aligned}
$$

where

$$
\begin{aligned}
& \left(\beta_{x n}^{e(s)}\right)^{2}=\epsilon_{r}^{(s)} k_{0}^{2}-\left(\beta_{y n}^{e(s)}\right)^{2}-\gamma^{2} \\
& \left(\beta_{x n}^{h(s)}\right)^{2}=\epsilon_{r}^{(s)} k_{0}^{2}-\left(\beta_{y n}^{h(s)}\right)^{2}-\gamma^{2} .
\end{aligned}
$$

Referring to Fig. 2(a), in (5a) and (5b), $x_{31}=0, x_{32}=r$, $x_{33}=r+p, x_{41}=0$, and $x_{42}=a$. Note that, in the slot regions $s=41$ and $42, \beta_{x n}^{e(s)}=\beta_{x n}^{h(s)}=n \pi / d_{(s)}$. We simply 
use the $x$-directed Hertzian potentials for the slot regions 41 and 42 , knowing that there is no perference for choosing either $x$ - or $y$-directed Hertzian potentials here. On the other hand $\phi_{n}^{e(s)}(x), \phi_{n}^{h(s)}(x), \psi_{n}^{e(s)}(y)$, and $\psi_{n}^{h(s)}(y)$ in the slot regions 41 and 42 have field expressions similar to those shown in (3a)-to-(4b), respectively. In region 3 , the coefficients $c_{n}^{e(s)}, d_{n}^{e(s)}, c_{n}^{h(s)}$, and $d_{n}^{h(s)}$ and eigenvalues $\beta_{x n}^{e(s)}$ and $\beta_{x n}^{h(s)}$ of (5a) and (5b) can be straightforwardly determined by matching the tangential boundary conditions at $x=x_{32}=r$ and $x=x_{33}=r+p$, respectively [27]. Note that $(5 a)$ and $(5 b)$ in region 3 satisfy the well-known biorthogonality relations

$$
\begin{array}{r}
\int_{0}^{a} \phi_{n}^{e(3)}(x) \phi_{m}^{e(3)}(x) / \epsilon(x) d x=\delta_{m}^{n} \\
\int_{0}^{a} \phi_{n}^{h(3)}(x) \phi_{m}^{h(3)}(x) d x=\delta_{m}^{n}
\end{array}
$$

where $\delta_{m}^{n}$ is the Kronecker delta function. The eigenfunction expansions in the $y$ direction are identical to those shown in (4a) and (4b).

\section{Boundary Conditions in the Horizontal Plane}

At interfaces $y=y_{1}, y=y_{2}$, and $y=y_{5}$, the tangential electric and magnetic fields should match and the boundary conditions are

$$
\begin{array}{lll}
E_{z}^{(s)}=E_{z}^{(s+1)} & E_{x}^{(s)}=E_{x}^{(s+1)}, & x \in[0, a] \\
H_{z}^{(s)}=H_{z}^{(s+1)} & H_{x}^{(s)}=H_{x}^{(x+1)}, & x \in[0, a]
\end{array}
$$

where $s=1,2$, and 5 . At the interface $y=y_{3(4)}$, the tangential electric and magnetic fields should match as follows:

$$
\begin{aligned}
& E_{z}^{4}=E_{z}^{3(5)} \quad E_{x}^{4}=E_{x}^{3(5)}, \quad x \in[0, a] \\
& E_{z}^{4}=E_{z}^{3(5)}=0 \quad E_{x}^{4}=E_{x}^{3(5)}=0, \quad x \in\left[d_{41}, d_{41}+w\right] \\
& H_{z}^{41}=H_{z}^{3(5)} \quad H_{x}^{41}=H_{x}^{3(5)}, \quad x \in\left[0, d_{41}\right] \\
& H_{z}^{42}=H_{z}^{3(5)} \\
& H_{x}^{42}=H_{x}^{3(5)} \text {, } \\
& x \in\left[d_{41}+w, a\right] \text {. }
\end{aligned}
$$

\section{Coefficient Elimination Process}

Fig. 2(b) depicts the setup of the corresponding coefficients of the eigenfunction expansions in subsections II-A and II-B. Although the coefficient elimination process in the classical [24] and the modified mode-matching methods [25], [26] are well known, the proposed mixed potentials mode-matching formulation requires special attention. In view of Fig. 2(b), one needs to eliminate the coefficients in order to reduce the matrix size of the final nonstandard eigenvalue equation for obtaining the value of the propagation constant $(\gamma)$. Many possible forms for the final nonstandard eigenvalue equation can exist. This implies that the nonstandard eigenvalue equation is not unique. We will recommend a systematic procedure in three steps for eliminating the coefficients of Fig. 2(b) to such an extent that the analytical handling of the coeffi- cient elimination process is not too involved and the use of mixed Hertzian potentials can be fully appreciated.

Step 1) Relating the Coefficients in the Layered Regions: By matching the boundary conditions (7a) and (7b) at $y=y_{1}$ and $y=y_{5}$, respectively, and invoking the orthogonality relations of the eigenfunctions $(3 \mathrm{a})$ and $(3 \mathrm{~b})$ in the layered regions 2 and 5 , one obtains the following expressions relating the corresponding coefficients, namely

$\begin{array}{ll}a_{n}^{e(2)}=-\frac{\epsilon_{1} \beta_{y n}^{(2)} \tan \left(\beta_{y n}^{(2)} t_{2}\right)}{\epsilon_{2} \beta_{y n}^{(1)} \tan \left(\beta_{y n}^{(1)} t_{1}\right)} b_{n}^{e(2)}, & n=1,2, \cdots, N_{2} \\ a_{n}^{h(2)}=\frac{\beta_{y n}^{(2)} \tan \left(\beta_{y n}^{(2)} t_{2}\right)}{\beta_{y n}^{(1)} \cot \left(\beta_{y n}^{(1)} t_{1}\right)} b_{n}^{h(2)}, & n=0,1,2, \cdots, N_{2}\end{array}$

$a_{n}^{e(5)}=-\frac{\epsilon_{6} \beta_{y n}^{(5)} \cot \left(\beta_{y n}^{(5)} t_{5}\right)}{\epsilon_{5} \beta_{y n}^{(6)} \tan \left(\beta_{y n}^{(6)} t_{6}\right)} b_{n}^{e(5)}, \quad n=1,2, \cdots, N_{5}$

$a_{n}^{h(5)}=\frac{\beta_{y n}^{(6)} \tan \left(\beta_{y n}^{(6)} t_{6}\right)}{\beta_{y n}^{(5)} \tan \left(\beta_{y n}^{(5)} t_{5}\right)} b_{n}^{h(5)}, \quad n=0,1,2, \cdots, N_{5}$.

The procedure outlined here is similar to that reported in [28] and analogous to the modified mode-matching methods [25], [26].

Step 2) Relating the Coefficients of the Layered and Nonlayered Regions: At $y=y_{2}$, in particular, the boundary conditions ( $7 \mathrm{a}$ ) and (7b) should be satisfied between the layered and nonlayered dielectric regions. The biorthogonality relations for region 3 , i.e., (6a) and (6b), are applied to the boundary conditions $H_{x}^{(s)}=H_{x}^{(s+1)}$ and $E_{x}^{(s)}=E_{x}^{(s+1)}$, where $s=2$, since $\phi_{n}^{e(3)}$ and $\psi_{n}^{h(3)}$ do not take any derivative with respect to $x$ for obtaining $E_{x}^{(3)}$ and $H_{x}^{(3)}$ when using the $x$-directed Hertzian potentials. After some algebraic manipulation and using (8a) and (8b) to further eliminate $a_{n}^{e(2)}$ and $a_{n}^{h(2)}$, the abbreviated expressions relating the coefficients in these regions are

$$
\begin{array}{ll}
a_{n}^{e(3)}=\sum_{k=1}^{N_{2}} A_{n k}^{1} b_{n}^{e(2)}+\sum_{k=0}^{N_{2}} A_{n k}^{2} b_{n}^{h(2)}, & n=0 \cdots N_{3} \\
a_{n}^{h(3)}=\sum_{k=1}^{N_{2}} B_{n k}^{1} b_{n}^{e(2)}+\sum_{k=0}^{N_{2}} B_{n k}^{2} b_{n}^{h(2)}, & n=1 \cdots N_{3} .
\end{array}
$$

Next the orthogonality relations for region 2 are applied to boundary conditions $E_{z}^{(s)}=E_{z}^{(s+1)}$ and $H_{z}^{(s)}=H_{z}^{(s+1)}$. Thus we obtain the following abbreviated expressions:

$$
\begin{array}{r}
F_{1 k} b_{k}^{e(2)}+F_{2 k} b_{k}^{h(2)}=\sum_{m=0}^{N_{3}} C_{k m}^{1} a_{m}^{e(3)}+\sum_{m=1}^{N_{3}} C_{k m}^{2} b_{m}^{h(3)}, \\
k=0 \cdots N_{2} \\
F_{3 k} b_{k}^{e(2)}+F_{4 k} b_{k}^{h(2)}=\sum_{m=0}^{N_{3}} D_{k m}^{1} b_{m}^{e(3)}+\sum_{m=1}^{N_{3}} D_{k m}^{2} a_{m}^{h(3)}, \\
k=0 \cdots N_{2}
\end{array}
$$


where $b_{0}^{e(2)}$ is equal to zero. Subsequently $b_{k}^{e(2)}$ and $b_{k}^{h(2)}$ can be solved in terms of $a_{m}^{e(3)}, b_{m}^{h(3)}, b_{m}^{e(3)}$, and $a_{m}^{h(3)}$ using (11a) and (11b). Substituting these results into (10a) and (10b) to eliminate $b_{k}^{e(2)}$ and $b_{k}^{h(2)}$, we obtain the following abbreviated expressions in terms of $a_{m}^{e(3)}, b_{m}^{e(3)}, a_{m}^{h(3)}$, and $b_{m}^{h(3)}$ :

$$
\begin{aligned}
a_{n}^{e(3)}= & \sum_{m=0}^{N_{3}} \sum_{l=1}^{N_{2}} G_{l m}^{l n} a_{m}^{e(3)}+\sum_{m=0}^{N_{3}} \sum_{l=0}^{N_{2}} G_{l m}^{2 n} b_{m}^{e(3)} \\
& +\sum_{m=1}^{N_{3}} \sum_{l=0}^{N_{2}} G_{l m}^{3 n} a_{m}^{h(3)}+\sum_{m=1}^{N_{3}} \sum_{l=1}^{N_{2}} G_{l m}^{4 n} b_{m}^{h(3)}, \\
& n=0 \cdots N_{3} \\
a_{n}^{h(3)}= & \sum_{m=0}^{N_{3}} \sum_{l=1}^{N_{2}} G_{l m}^{5 n} a_{m}^{e(3)}+\sum_{m=0}^{N_{3}} \sum_{l=0}^{N_{2}} G_{l m}^{6 n} b_{m}^{e(3)} \\
& +\sum_{m=1}^{N_{3}} \sum_{l=0}^{N_{2}} G_{l m}^{7 n} a_{m}^{h(3)}+\sum_{m=1}^{N_{3}} \sum_{l=1}^{N_{2}} G_{l m}^{8 n} b_{m}^{h(3)}, \\
n=1 & \cdots N_{3} .
\end{aligned}
$$

Step 3) Relating the Coefficients of the Slot Regions and the Adjacent Layered and Nonlayered Regions: Matching the tangential boundary conditions (7c) through (7f) at $y=y_{4}$ and applying the orthogonality relations in slot and layered regions, respectively, one obtains four sets of equations. By using (9a) and (9b) one may eliminate $a_{n}^{e(5)}$ and $a_{n}^{h(5)}$. Next $b_{n}^{e(5)}$ and $b_{n}^{h(5)}$ are solved in terms of the slot coefficients using two of the four equations. Substituting these results into the remaining two equations, one obtains the following abbreviated expressions in terms of the slot coefficients:

$$
\begin{aligned}
& k_{5 n}^{(s)} a_{n}^{e(s)}+k_{6 n}^{(s)} b_{n}^{e(s)}=\sum_{m=0}^{N_{41}}\left(\sum_{l=0}^{N_{5}} H_{l m}^{1 n(s)}+\sum_{l=1}^{N_{5}} \overline{\bar{H}}_{l m}^{l n(s)}\right) a_{m}^{e(41)} \\
& +\sum_{m=0}^{N_{41}}\left(\sum_{l=0}^{N_{5}} H_{l m}^{2 n(s)}+\sum_{l=1}^{N_{5}} \overline{\bar{H}}_{l m}^{2 n(s)}\right) b_{m}^{e(41)} \\
& +\sum_{m=1}^{N_{41}}\left(\sum_{l=0}^{N_{5}} H_{l m}^{3 n(s)}+\sum_{l=1}^{N_{5}} \overline{\bar{H}}_{l m}^{3 n(s)}\right) a_{m}^{h(41)} \\
& +\sum_{m=1}^{N_{41}}\left(\sum_{l=0}^{N_{5}} H_{l m}^{4 n(s)}+\sum_{l=1}^{N_{\varsigma}} \overline{\bar{H}}_{l m}^{4 n(s)}\right) b_{m}^{h(41)} \\
& +\sum_{m=0}^{N_{42}}\left(\sum_{l=0}^{N_{5}} H_{l m}^{5 n(s)}+\sum_{l=1}^{N_{5}} \overline{\bar{H}}_{l m}^{5 n(s)}\right) a_{m}^{e(42)} \\
& +\sum_{m=0}^{N_{42}}\left(\sum_{l=0}^{N_{5}} H_{l m}^{6 n(s)}+\sum_{l=1}^{N_{5}} \overline{\bar{H}}_{l m}^{6 n(s)}\right) b_{m}^{e(42)} \\
& +\sum_{m=1}^{N_{42}}\left(\sum_{l=0}^{N_{5}} H_{l m}^{7 n(s)}+\sum_{l=1}^{N_{5}} \overline{\bar{H}}_{l m}^{7 n(s)}\right) a_{m}^{h(42)} \\
& +\sum_{m=1}^{N_{42}}\left(\sum_{l=0}^{N_{5}} H_{l m}^{8 n(s)}+\sum_{l=1}^{N_{5}} \overline{\bar{H}}_{l m}^{8 n(s)}\right) b_{m}^{h(42)}, \\
& n=0 \cdots N_{(s)}
\end{aligned}
$$

$$
\begin{aligned}
& a_{n}^{h(s)}+b_{n}^{h(s)}=\sum_{m=0}^{N_{41}}\left(\sum_{l=0}^{N_{5}} H_{l m}^{1 n(s)}+\sum_{l=1}^{N_{5}} \overline{\bar{H}}_{l m}^{l n(s)}\right) a_{m}^{e(41)} \\
& +\sum_{m=0}^{N_{41}}\left(\sum_{l=0}^{N_{5}} H_{l m}^{2 n(s)}+\sum_{l=1}^{N_{5}} \overline{\bar{H}}_{l m}^{2 n(s)}\right) b_{m}^{e(41)} \\
& +\sum_{m=1}^{N_{41}}\left(\sum_{l=0}^{N_{5}} H_{l m}^{3 n(s)}+\sum_{l=1}^{N_{5}} \overline{\bar{H}}_{l m}^{3 n(s)}\right) a_{m}^{h(41)} \\
& +\sum_{m=1}^{N_{41}}\left(\sum_{l=0}^{N_{5}} H_{l m}^{4 n(s)}+\sum_{l=1}^{N_{5}} \overline{\bar{H}}_{l m}^{4 n(s)}\right) b_{m}^{h(41)} \\
& +\sum_{m=0}^{N_{42}}\left(\sum_{l=0}^{N_{5}} H_{l m}^{5 n(s)}+\sum_{l=1}^{N_{5}} \overline{\bar{H}}_{l m}^{5 n(s)}\right) a_{m}^{e(42)} \\
& +\sum_{m=0}^{N_{42}}\left(\sum_{l=0}^{N_{5}} H_{l m}^{6 n(s)}+\sum_{l=1}^{N_{5}} \overline{\bar{H}}_{l}^{6 n(s)}\right) b_{m}^{e(42)} \\
& +\sum_{m=1}^{N_{42}}\left(\sum_{l=0}^{N_{5}} H_{l m}^{7 n(s)}+\sum_{l=1}^{N_{5}} \overline{\bar{H}}_{l m}^{7 n(s)}\right) a_{m}^{h(42)} \\
& +\sum_{m=1}^{N_{42}}\left(\sum_{l=0}^{N_{5}} H_{l m}^{8 n(s)}+\sum_{l=1}^{N_{5}} \overline{\bar{H}}_{l m}^{8 n(s)}\right) b_{m}^{h(42)}, \\
& n=1 \cdots N_{(s)} \quad s=41,42 .
\end{aligned}
$$

Note that (13a) and (13b) represent four sets of equations since $s=41$ and 42 . At the interface $y=y_{3}$ the treatment is different from the procedure matching the boundary conditions at $y=y_{4}$ since $d \phi_{n}^{e(3)} / d x$ or $d \phi_{n}^{h(3)} / d x$ terms exist in the expression for $E_{z}^{(3)}$ and $H_{z}^{(3)}$ and no crossorthogonality relations exist between $\phi_{n}^{e(3)}, \phi_{n}^{h(3)}$, $d \phi_{n}^{e(3)} / d x$, and $d \phi_{n}^{h(3)} / d x$. Using the boundary conditions (7e) and (7f) and applying the orthogonality relations applied in regions 41 and 42 , one obtains

$$
\begin{aligned}
& a_{n}^{h(s)}=\sum_{m=1}^{N_{3}} I_{n m}^{1(s)} a_{m}^{h(3)}+ \sum_{m=1}^{N_{3}} I_{n m}^{2(s)} b_{m}^{h(3)}, \quad n=1 \cdots N_{(s)} \\
& k_{7 n} a_{n}^{h(s)}+k_{8 n} b_{n}^{e(s)}= \sum_{m=0}^{N_{3}} I_{n m}^{3(s)} a_{m}^{e(3)}+\sum_{m=0}^{N_{3}} I_{n m}^{4(s)} b_{m}^{e(3)} \\
&+\sum_{m=1}^{N_{3}} I_{n m}^{5(s)} a_{m}^{h(3)}+\sum_{m=1}^{N_{3}} I_{n m}^{6(s)} b_{m}^{h(3)} \\
& s=41,42 \quad\left(a_{0}^{h(s)}=0\right) \quad n=0 \cdots N_{(s)} .
\end{aligned}
$$

By satisfying boundary conditions (7c) and (7d) and apply- 
ing biorthogonality relations (6a) and (6b), one obtains

$$
\begin{gathered}
a_{n}^{e(3)}+b_{n}^{e(3)}=\sum_{m=0}^{N_{41}} J_{n m}^{1} a_{m}^{e(41)}+\sum_{m=0}^{N_{42}} J_{n m}^{2} a_{m}^{e(42)}, \\
n=0 \cdots N_{3} \\
k_{9 n} a_{n}^{h(3)}+k_{10 n} b_{n}^{h(3)}+\sum_{m=0}^{N_{3}} L_{n m}^{1} a_{m}^{e(3)}+\sum_{m=0}^{N_{3}} L_{n m}^{2} b_{m}^{e(3)} \\
=\sum_{m=0}^{N_{41}} L_{n m}^{3} a_{m}^{e(41)}+\sum_{m=1}^{N_{41}} L_{n m}^{4} b_{m}^{h(41)}+\sum_{m=0}^{N_{42}} L_{n m}^{5} a_{m}^{e(42)} \\
+\sum_{m=1}^{N_{42}} L_{n m}^{6} b_{m}^{h(42)}, \quad n=1 \cdots N_{3} .
\end{gathered}
$$

Grouping the expressions (12a), (12b), (13a), (13b), (14a), (14b), (15a), and (15b), one finally obtains the following nonstandard eignevalue equation for solving the propagation constant $(\gamma)$ :

$$
[A(\gamma)][x]=[0]
$$

where the square matrix [ $A$ ] has the dimension of $4\left(N_{3}+\right.$ $\left.N_{41}+N_{42}\right)+6$ by $4\left(N_{3}+N_{41}+N_{42}\right)+6$. The nontrivial solution for the column vector $x$ requires that $\operatorname{det}(A(\gamma))=0$. When the value of the propagation constant $(\gamma)$ is obtained, the electromagnetic field solution is solved by knowing the column vector $x$, which is within a constant multiplicative factor.

\section{Convergence Study of the Proposed Full-Wave Method}

To obtain the solutions for the propagation constant $(y)$ in the matrix equation (16), one needs to truncate the matrix equation by using a finite number of eigenfunction expansion terms in each region. As pointed out by various authors [5], [26], [27], [29], [30], using basically the modematching method in different problems, the relative convergence criterion should be satisfied to obtain (1) good field matching at metal-dielectric interfaces [5], [26], [27]; (2) fast converged susceptance values for iris-type discontinuity problems [29]; and (3) an accurate solution for the reflection coefficient in microstrip step-discontinuity problems [30], to name a few. It is plausible to speculate that the proposed mixed potential mode-matching method may encounter similar convergence problems.

Throughout this paper, the theoretical data associated with Fig. 2(a) are presented. Fig. 2(a) consists of a perfect metal strip of finite thickness, a nonlayered region with two dielectric step discontinuities located at $x=r$ and $x=r+p$, respectively, and some layered regions. Note that, as shown in parts (a)-(c) of Figs. 3, the microstrip substrate either touches or separates from the sidewalls of the perfectly conducting electric enclosure. By setting the values of $t_{1}$ and $t_{2}$ in Fig. 2(a) equal to zero, a finite-width microstrip configuration is obtained. Using $N_{5}\left(N_{6}\right)$ and $N_{41}\left(N_{42}\right)$ as the controlling parameters, parts (a)-(c) of Figs. 3 illustrate the convergence properties of the three different cases for a symmetric microstirp with $p=10 w$,

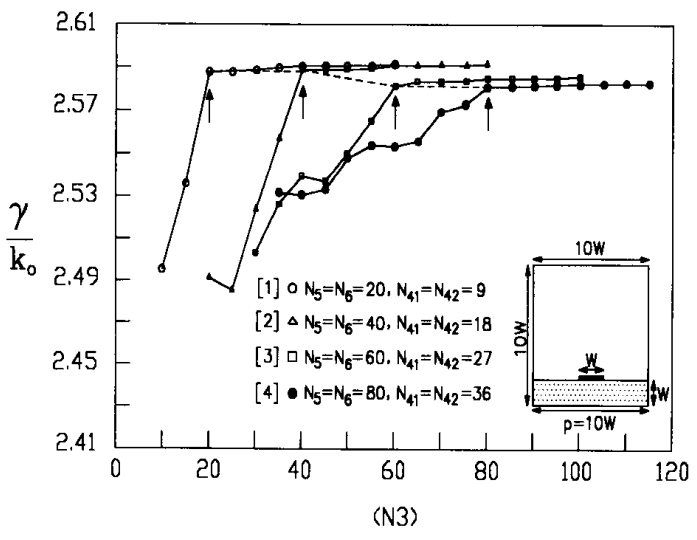

(a)

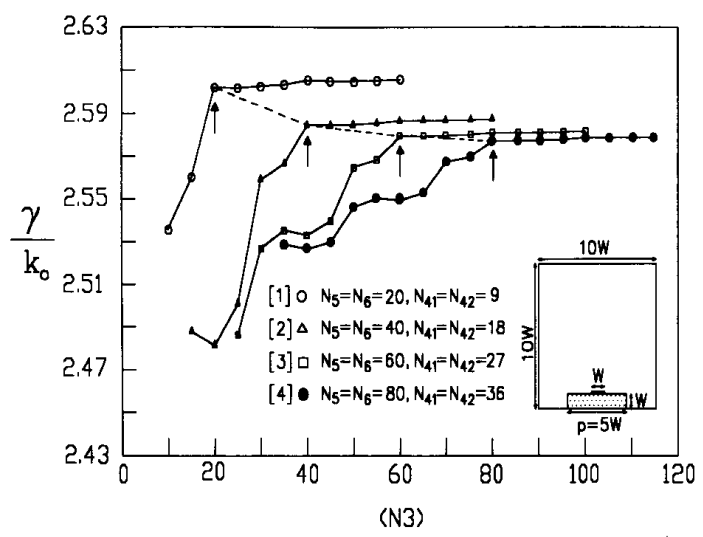

(b)

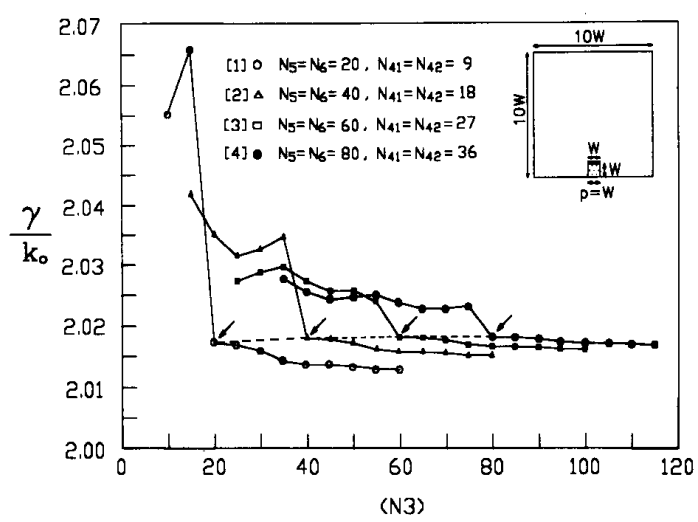

(c)

Fig. 3. Relative convergence studies for the normalized propagation constant $\left(\gamma / k_{0}\right)$ of the fundamental mode for a shielded symmetric microstrip line at $10 \mathrm{GHz}$ versus the value of $N_{3}$ for three case studies using different substrate widths. The arrow head points to the position where the relative convergence criterion is satisfied for each family of curves. Structural parameters corresponding to Fig. 2(a) are $a=b=12.7$ $\mathrm{mm}, t_{1}=t_{2}=0, t_{3}=1.27 \mathrm{~mm}, t_{4}=0.01 \mathrm{~mm}, t_{5}+t_{6}=11.42 \mathrm{~mm}, w=1.27$ $\mathrm{mm}, d_{41}=d_{42}=5.715 \mathrm{~mm}, s_{1}=s, r=q, \epsilon_{32}=8.875$, others $\epsilon_{r}=1.0$. (a) $p=10 w$. (b) $p=5 w$. (c) $p=w$. 


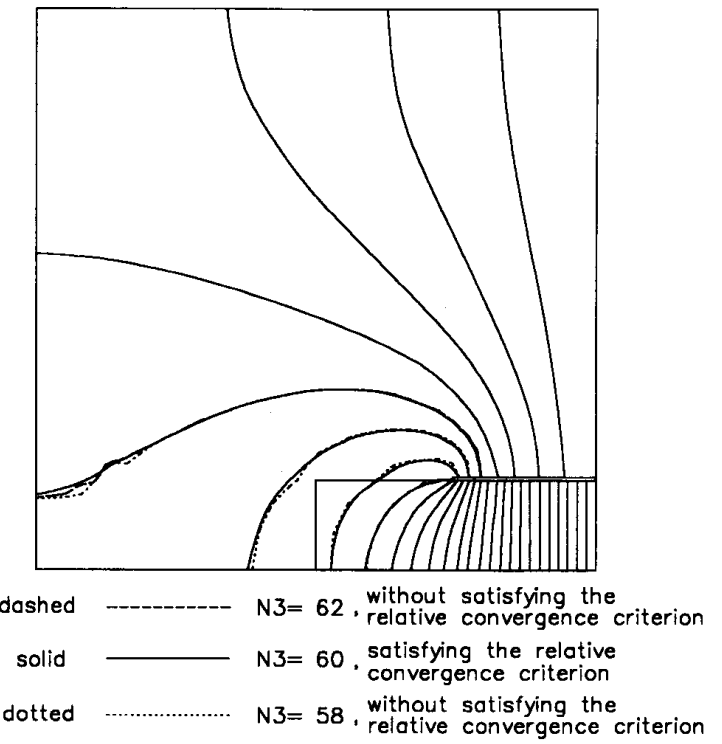

Fig. 4. The superimposed dominant mode transverse electric field patterns of a symmetric microstrip enclosed by a WR-12 waveguide housing under conditions satisfying and not satisfying the relative convergence criterion. Structural parameters corresponding to Fig. 2(a) are $w=0.775 \mathrm{~mm}, a=4 w=3.1 \mathrm{~mm}, b=2 w=1.55 \mathrm{~mm}, t_{1}=t_{2}=0, t_{3}=$ $0.254 \mathrm{~mm}, t_{4}=0.005 \mathrm{~mm}, t_{5}+t_{6}=1.291 \mathrm{~mm}, d_{41}=d_{42}=1.1625 \mathrm{~mm}$ $r=q=0.775 \mathrm{~mm}, p=2 w=1.55 \mathrm{~mm}, \epsilon_{32}=2.2$, others $\epsilon_{r}=1.0, f=1.0$ GHz, $N_{5}=N_{6}=60, N_{41}=N_{42}=\left(d_{41} / a\right) 60=23$.

$5 w$, and $w$, respectively, where $p$ is the substrate width and $w$ is the width of the metal strip. Clearly the relative convergence problem exists in the new formulation since the normalized propagation constants of the three cases converge to different values as the value of $N_{3}$, the number of eigenfunction expansion terms in the nonlayered region, increases.

The numbers of the eigenfunction expansion terms used in regions 1 through 6 are denoted $N_{1}, N_{2}, N_{3}, N_{41}$, $N_{42}, N_{5}$, and $N_{6}$, respectively. Since the relative convergence criterion for the interface between the metal and layered dielectric is already established [5], [26], [27], [29], [30], the ratio of $N_{5}\left(N_{5}=N_{6}\right)$ to $N_{41}\left(N_{41}=N_{42}\right)$ is kept equal to the aspect ratio, i.e., $10 w / 4.5 w\left(a / d_{41}\right)$, in parts (a) through (c) of Fig. 3. In Fig. 3(a), the arrow heads point to the locations where the ratio of $N_{3}$ to $N_{41}\left(N_{3}\right.$ to $N_{42}$ ) is equal to the aspect ratio of $a$ to $d_{41}\left(a\right.$ to $\left.d_{42}\right)$. These points represent the condition whereby the relative convergence criterion is met at the interface between metal and nonlayered regions. The loci of these points, connected by the dashed line, depict a smooth and rapidly convergent property similar to the case of layered substrates reported earlier [5], [27]. Here the same smooth loci satisfying the relative convergence criterion for both interfaces at metal-layered regions and metal-nonlayered regions manifest the fact that the proposed formulation also mandates the relative convergence criterion as discussed before [5], [26], [27], [29], [30].

In addition to the supporting evidence for the necessity of invoking the relative convergence criterion illustrated

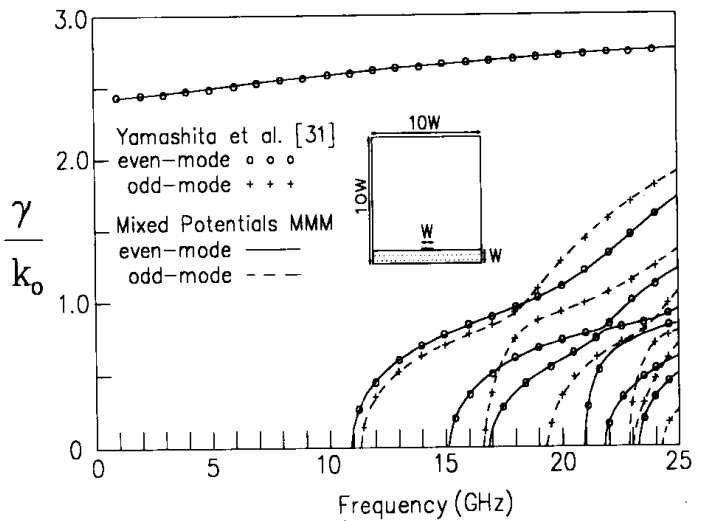

Fig. 5. Normalized propagation constant $\left(\gamma / k_{0}\right)$ versus frequency for a shielded microstrip obtained by the proposed mixed potential modematching method and the nonuniform discretization integral equations [31]. Structural parameters corresponding to Fig. 2(a) are $a=b=12.7$ $\mathrm{mm}, t_{1}=t_{2}=0, t_{3}=1.27 \mathrm{~mm}, t_{4}=0.01 \mathrm{~mm}, t_{5}+t_{6}=11.42 \mathrm{~mm}, w=1.27$ $\mathrm{mm}, d_{41}=d_{42}=5.715 \mathrm{~mm}, r=q=0, p=12.7 \mathrm{~mm}, \epsilon_{32}=8.875$, others $\epsilon_{r}=1.0, N_{3}=25, N_{41}=N_{42}=11, N_{5}=N_{6}=25$.

in parts (a)-(c) of Figs. 3 for good electromagnetic field solutions, Fig. 4 superimposes three transverse electric field patterns of a finite-width symmetric microstrip line under various conditions satisfying and not satisfying the relative convergence criterion. According to the parameters given in Fig. 4, the solid line case with $N_{3}=60$ satisfies the relative convergence criterion. By offsetting only two terms from $N_{3}=60$, the resultant electric field patterns are in dashed and dotted lines. Clearly they exhibit some irregularities near the bottom left corner of Fig. 4. Only the solid line, obtained by satisfying the relative convergence criterion, shows the smoothest electric field lines throughout the entire waveguide cross section. Therefore, to obtain very accurate electromagnetic guided-wave solutions based on the proposed formulation, the relative convergence criterion must be satisfied.

\section{Proximity Effects on Microstrip near Substrate Edge and Sidewall}

One application of the proposed formulation is the investigation of proximity effects of microstrip and Microslab. Before presenting the data for the proximity effects, we need to validate the new formulation by comparing the theoretic results with the available data.

\section{A. Validity Check of the Proposed Formulation}

Fig. 5 plots the dispersion characteristics of the fundamental and higher-order modes from 1 to $25 \mathrm{GHz}$ for a shielded symmetric microstrip with a layered substrate. The even- and odd-mode data reported by Yamashita et al. [31] are denoted by $\operatorname{circle}(\circ)$ and plus $(+)$ symbols, respectively. The solid and dashed lines are for the evenand odd-mode data obtained by the present mixed potential mode-matching method satisfying the relative convergence criterion. The value of $w / a$ ratio is equal to 0.1 


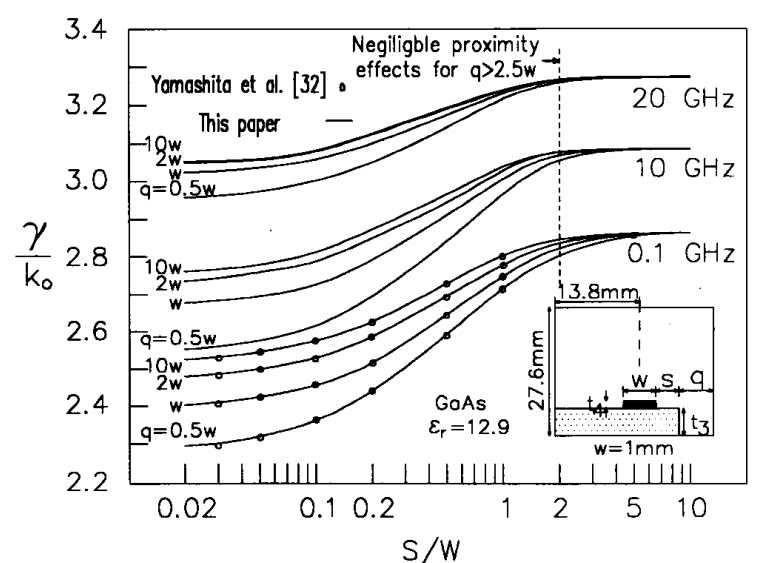

Fig. 6. The proximity effects of sidewall and one substrate edge on the fundamental mode normalized propagation constant $\left(\gamma / k_{0}\right)$ by varying the distance $q$ between the sidewall and substrate edge and the distance $s$ between the edges of microstrip and substrate at three different frequencies. The data under the circle symbol are based on the quasiTEM approach [32]. Structural parameters corresponding to Fig. 2(a) are $w=1.0 \mathrm{~mm}, b=27.6 \mathrm{~mm}, t_{1}=t_{2}=0, t_{3}=1.382 \mathrm{~mm}, t_{4}=1.0 \mu \mathrm{m}$, $t_{5}+t_{6}=26.217 \mathrm{~mm}, r=0, p=14.3 \mathrm{~mm}+s, s_{1}=13.3 \mathrm{~mm}, a=14.3$ $\mathrm{mm}+s+q, N_{3}=N_{5}=N_{6}=100, N_{41}=\left(d_{41} / a\right) N_{3}, N_{42}=\left(d_{42} / a\right) N_{3}$.

and the finite conductor thickness is $1 \mu \mathrm{m}$. Fig. 5 shows that excellent agreement is obtained for the published data and those obtained by the proposed formulation.

\section{B. Microstrip Proximity Effects}

Fig. 6 shows the proximity effects of sidewall and substrate edge on the normalized propagation constant of an asymmetric microstrip. The normalized propagation constant is plotted against the spacing $s$, defined as the distance between the edges of the metal strip and dielectric substrate, using the distance $q$, defined as the distance between the sidewall and substrate edge, as the controlling parameter at three different frequencies. Note that we will use the same terminology for $s$ and $q$ in the next section studying the proximity effects of Microslab. Both $s$ and $q$ are normalized with respect to the width, $w$, of the metal strip. A GaAs substrate is assumed. The line width, $w$, and the substrate thickness, $t_{3}$, are chosen so that a $50 \Omega$ line operating at a much lower frequency is established when the proximity effect is negligible. In the low-frequency limit, i.e. $0.1 \mathrm{GHz}$, the data obtained by the proposed method are in excellent agreement with those reported by Yamashita et al. using a quasi-TEM approach [32]

Given a fixed $s / w$ ratio in Fig. 6 and varying the distance $q$ from $0.5 w$ to $10 w$, the normalized propagation constant increases. The case with a higher value of $q$ means that the sidewall is moved farther. This results in less electromagnetic field energy in the air between sidewall and substrate edge. Therefore the propagation constant increases. When the value of the $s / w$ ratio increases to more than 5 at a given frequency, all the normalized propagation constants with respect to different values of $q$, namely, $0.5 w, w, 2 w$, and $10 w$, approach

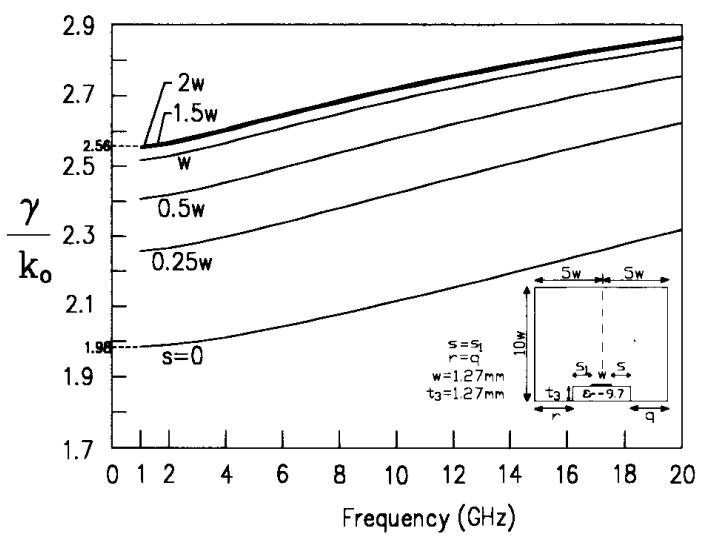

Fig. 7. The proximity effects of sidewalls and two substrate edges on the fundamental mode normalized propagation constant $\left(\gamma / k_{0}\right)$ of a shielded symmetric microstrip line by equally varying the distances $s$ and $s_{1}$ between the edges of microstrip and substrate. Structural and material parameters corresponding to Fig. 2(a) are $a=b=12.7 \mathrm{~mm}$, $t_{1}=t_{2}=0, t_{3}=1.27 \mathrm{~mm}, t_{4}=0.01 \mathrm{~mm}, t_{5}+t_{6}=11.42 \mathrm{~mm}, w=1.27$ $\mathrm{mm}, d_{41}=d_{42}=5.715 \mathrm{~mm}, s_{1}=s, p=w+2 s, r=q, \epsilon_{32}=9.7$, others $\epsilon_{r}=1.0, N_{3}=N_{5}=N_{6}=40, N_{41}=N_{42}=18$.

the same value. This reflects the fact that the proximity effects can be negligible when the $s / w$ ratio is greater than 5 for $q \geqslant 0.5 w$. On the other hand, when the $s / w$ ratio decreases, the microstrip is closer to the edge of the $\mathrm{GaAs}$ substrate this makes the proximity effects of the microstrip line highly subject to the location of the sidewall, since the electromagnetic field coupling between the sidewall and the microstrip line near the substrate edge have increased substantially.

Given a fixed value of $s / w$ ratio and varying the value of $q$ from $0.5 w$ to $10 w$, Fig. 6 also shows that the deviation of the normalized propagation constants decreases as frequency increases from 0.1 to $20 \mathrm{GHz}$. Conversely, given a fixed value of $q$ and varying the $s / w$ ratio from 0.02 to 10 , the deviation of the normalized propagation constants decreases as frequency increases from 0.1 to $20 \mathrm{GHz}$. Thus the effects of the sidewall and substrate edge, or the proximity, become less significant at higher frequencies. Again, these observations reflect the fact that the electromagnetic field tends to be confined underneath the metal strip at higher frequencies. Consequently any further structural variations in $s$ and $q$ will cause smaller perturbations in the electromagnetic field distribution at higher frequencies and result in less deviation of the propagation constants compared with the lower frequency data.

Next we investigate the proximity effects of double substrate edges on a symmetric microstrip. Fig. 7 plots the fundamental mode normalized propagation constant of a shielded symmetric microstrip line against frequency while simultaneously varying the value of $s$ and $s_{1}\left(s=s_{1}\right)$. When $s=0$, the substrate edges line up with the metal strip edges. The normalized propagation constant changes drastically when the value of $s$ increases by a small amount from $s=0$. Note that when $s$ varies the substrate 


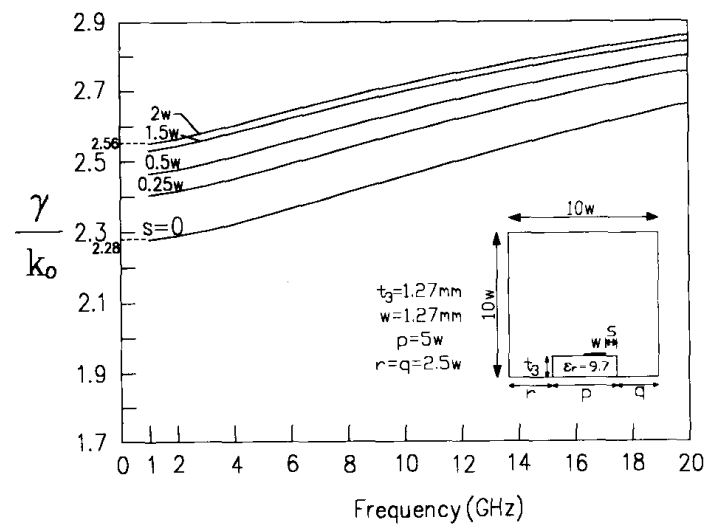

Fig. 8. The proximity effects of sidewalls and two substrate edges on the fundamental mode normalized propagation constant $\left(\gamma / k_{0}\right)$ of a shielded asymmetric microstrip line with fixed finite-width substrate. Structural and material parameters corresponding to Fig. 2(a) are $a=$ $b=12.7 \mathrm{~mm}, t_{1}=t_{2}=0, t_{3}=1.27 \mathrm{~mm}, t_{4}=0.01 \mathrm{~mm}, t_{5}+t_{6}=11.42 \mathrm{~mm}$, $w=1.27 \mathrm{~mm}, p=6.35 \mathrm{~mm}=5 w, r=q=3.175 \mathrm{~mm}, \epsilon_{32}=9.7$, others $\epsilon_{r}=1.0, N_{3}=N_{5}=N_{6}=40, N_{41}=\left(d_{41} / a\right) N_{3}, N_{42}=\left(d_{42} / a\right) N_{3}$.

width, $p$, changes accordingly. When the value of $s$ is greater than $2 w$, the normalized propagation constant remains nearly unchanged for further increases in $s$. Note that when $s=2 w, q=2.5 w$. This implies that we may place the microstrip $2 w$ away from the substrate edge with $q \geqslant 2.5 w$ without changing the propagation characteristics in the particular case study. Turning to Fig. 6 again, the proximity effects can be negligible when $q \geqslant$ $2.5 w$ for $s \geqslant 2 w$.

Fig. 8 illustrates results that are essentially an extension of Fig. 7. The same structural and material parameters are used in the analysis except that the substrate width, $p$, is fixed and the metal strip is placed in different locations measured from the substrate edge. It is interesting to note that, at $f=1 \mathrm{GHz}$, the variation of the normalized propagation constant for $s=0$ to $2 w$ is nearly half that of Fig. 7. In Fig. 7 two substrate edges are close to the metal strip, whereas only one substrate edge is near the metal strip in Fig. 8. Thus the proximity effects almost double in Fig. 7 in the low-frequency limit.

\section{Microslab Proximity Effects}

The Microslab configuration discussed in this section is slightly different from that reported by Young and Itoh [12]. The modified structure shown in Figs. 9 and 10 takes into account the finite thickness of the metal strip and the extended dielectric substrate supporting the metal strip. There are at least two possible situations needed for analyzing the modified structure of Microslab. First, it is likely that, in practice, the supporting dielectric substrate will extend beyond the edge of the metal strip during the fabrication phase. Second, the modified Microslab waveguide may offer a greater degree of freedom in use. Fig. 9 shows the effect of an extended supporting dielectric substrate on the normalized propagation constant versus frequency with the structure shown at the upper left

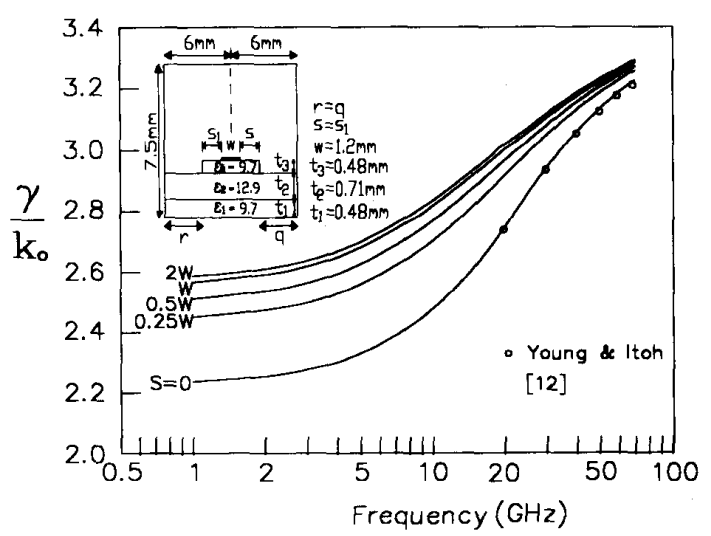

Fig. 9. Proximity effects of the extended supporting dielectric substrate on the dominant mode normalized propagation constant $\left(\gamma / k_{0}\right)$ of a shielded symmetric modified Microslab when varying $s$. Structural and material parameters corresponding to Fig. 2(a) are $a=12 \mathrm{~mm}$, $b=7.5 \mathrm{~mm}, w=1.2 \mathrm{~mm}, d_{41}=d_{42}=5.4 \mathrm{~mm}, t_{1}=0.48 \mathrm{~mm}, t_{2}=0.71$ $\mathrm{mm}, t_{3}=0.48 \mathrm{~mm}, t_{4}=1 \mu \mathrm{m}, t_{5}+t_{6}=5.829 \mathrm{~mm}, s_{1}=s, q=r, p=w+$ $2 s, \epsilon_{1}=9.7, \epsilon_{2}=12.9, \epsilon_{32}=9.7$, others $\epsilon_{r}=1, N_{1}=N_{2}=N_{3}=N_{5}=N_{6}$ $=40, N_{41}=N_{42}=18$.

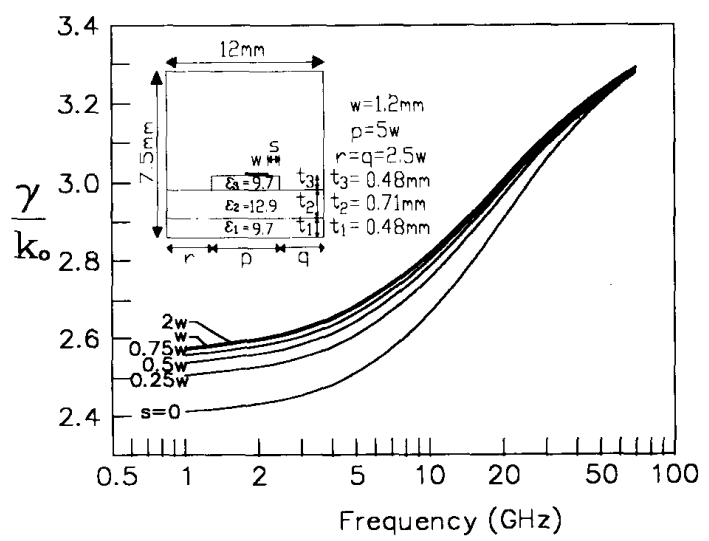

Fig. 10. Proximity effects of the extended dielectric substrate on the dominant mode normalized propagation constant $\left(\gamma / k_{0}\right)$ of a shielded asymmetric modified Microslab when varying $s$. Structural and material parameters corresponding to Fig. 2(a) are $a=12 \mathrm{~mm}, b=7.5 \mathrm{~mm}$, $w=1.2 \mathrm{~mm}, t_{1}=0.48 \mathrm{~mm}, t_{2}=0.71 \mathrm{~mm}, t_{3}=0.48 \mathrm{~mm}, t_{4}=1 \mu \mathrm{m}$, $t_{5}+t_{6}=5.829 \mathrm{~mm}, r=q=3 \mathrm{~mm}, p=6 \mathrm{~mm}=5 w, \epsilon_{1}=9.7, \epsilon_{2}=12.9$, $\epsilon_{32}=9.7$, others $\epsilon_{r}=1.0, \quad N_{1}=N_{2}=N_{3}=N_{5}=N_{6}=40, \quad N_{41}=$ $\left(d_{41} / a\right) N_{3}, N_{42}=\left(d_{42} / a\right) N_{3}$.

corner of the figure. In the particular case study, when $s$ and $s_{1}$ are equal to zero, the edges of the metal strip line up with the edges of the supporting dielectric substrate. The structure reduces to what was reported in [12] except that the finite metal thickness is considered here. Two sets of data, one obtained from the present method in solid line and the other from Young and Itoh [12] denoted by circles, agree with each other between 20 and 70 $\mathrm{GHz}$. (No data are available from [12] below $20 \mathrm{GHz}$.)

By simultaneously changing the values of $s$ and $s_{1}$ $\left(s=s_{1}\right)$ from 0 to $2 w$, the dispersion curves move upward. This implies that the higher the value of $s$, the less dispersive the propagation characteristics of the modified 
Microslab. The proximity effects from the extended supporting dielectric substrate are less significant at higher millimeter-wave frequency than at very low frequency for the particular structure under analysis.

Fig. 10 shows the proximity effects of the extended supporting dielectric substrate on the dominant mode normalized propagation constant of a shielded asymmetric Microslab. As the metal strip moves toward the edge of the supporting dielectric substrate, the value of $s$ changes from $2 w$ to 0 . Similar to the results illustrated in Fig. 9, the variation of the normalized propagation constants at $1 \mathrm{GHz}$ are much greater than at $70 \mathrm{GHz}$ when $s$ changes from 0 to $2 w$. The influence of the proximity effects of the substrate edge on the propagation constant is also less significant at higher frequencies. As a result, the Microslab, whether symmetric or asymmetric, is more subject to the proximity effects in the lower frequency region.

\section{Conclusion}

This paper proposes and validates the full-wave mixed potential mode-matching method for the full-wave analyses of a class of quasi-planar transmission lines containing layered and nonlayered substrates and finite metal thickness. The formulation of such guided-wave structures is derived in a systematic and easily implemented way. Extensive study of the convergence property of the new formulation is conducted and the results indicate that the relative convergence criterion needs to be satisfied for very accurate electromagnetic field solutions. This leads to a faster convergence property desired for most CAD (computer-aided design) modeling since the size of the nonstandard eigenvalue equation is reduced by using fewer expansion terms. By only offsetting two expansion terms from the required relative convergence criterion for a particular case study, some irregularities appear in the transverse electric field patterns of a microstrip on a finite-width substrate.

The validity of the proposed method is also confirmed by checking its results against the available published data for microstrip and Microslab, which are the reduced geometries of the particular case study shown in Fig. 2(a). Finally the dispersion characteristics of the proximity effects are investigated for the microstrip and Microslab. The physical implications of such data are discussed in detail.

\section{ACKNOWLEDGMENT}

The authors wish to thank the keen-eyed anonymous reviewers for their valuable suggestions and comments, which have made this paper clearer in its final form.

\section{REFERENCES}

[1] A. K. Sharma, "Considerations in producibility engineering of MMIC's" IEEE Trans. Microwave Theory Tech., vol. 38, pp. 1242-1248, Sept. 1990.
[2] T. Sueta and M. Izutsu, "Integrated optlc devices for microwave applications," IEEE Trans. Microwave Theory Tech., vol. 38, pp. 477-482, May 1990.

[3] R. H. Jansen, R. G. Arnold, and I. G. Eddison, "A comprehensive CAD approach to the design of MMIC's up to mm-wave frequencies," IEEE Trans. Microwave Theory Tech., vol. 36, pp. 208-219, Feb. 1988.

[4] T. Kitazawa and R. Mittra, "Analysis of finline with finite metallization thickness," IEEE Trans. Microwave Theory Tech., vol. MTT-32, pp. 1484-1487, Nov. 1984.

[5] W.-K. Wang, C.-K. C. Tzuang, J.-S. Chang, and T.-H. Wang, "Investigations of complex modes in a generalized bilateral finline with mounting grooves and finite conductor thickness," IEEE Trans. Microwave Theory Tech., vol. 37, pp. 1891-1897, Dec. 1989.

[6] M. Dagenais, R. F. Leheny, H. Temkin, and P. Bhattacharya "Applications and challenges of OEIC technology: A report on the 1989 Hilton head workshop," J. Lightwave Technol., vol. 8, pp. 846-861, June 1990.

[7] I. Kim, M. R. T. Tan, and S.-Y. Wang, "Analysis of a new microwave low-loss and velocity-matched III-V transmission line for traveling-wave electrooptic modulators," J. Lightwave Technol., vol. 8, pp. 728-738, May 1990.

[8] R. Delrue, E. Paleczny, J. F. Legier, P. Pribetich, and P. Kennis, "Microwave characteristics of planar electrooptic modulator," in IEEE MTT-S Int. Microwave Symp. Dig. (Long Beach, CA), 1989, pp. 1171-1174.

[9] E. Yamashita, K. Atsuki, and T. Akamatsu, "Application of microstrip analysis to the design of a broad-band electrooptical modulator," IEEE Trans. Microwave Theory Tech., vol. MTT-22, pp. 462-464, Apr. 1974.

[10] L. T. Yuan, "A W-band monolithic GaAs crossbar mixer," in IEEE Microwave and Millimeter-wave Monolithic Circuits Symp. Dig., 1984, pp. $67-69$.

[11] B. Young and T. Itoh, "Loss reduction in superconducting microstrip-like transmission lines," in IEEE MTT-S Int. Microwave Symp. Dig. (New York, NY), 1988, pp. 453-456.

[12] B. Young and T. Itoh, "Analysis and design of Microslab waveguide," IEEE Trans. Microwave Theory Tech., vol. MTT-35, pp. $850-857$, Sept. 1987.

[13] B. Roth, J. Borkes, M. Joseph, and A. Beyer, "A method for the development of monolithical integrated resonators on GaAs with increased $Q$-factor," in Proc. 20th European Microwave Conf. (Budapest), 1990, pp. 965-970.

[14] R. A. Pucel, "MMICs, modelling, and CAD-Where do we go from here?" in Proc. 16th European Microwave Conf. (Dublin), 1986, pp. $61-70$.

[15] D. G. Corr and J. B. Davies, "Computer analysis of the fundamental and higher order modes in single and coupled microstrip," IEEE Trans. Microwave Theory Tech., vol. MTT-20, pp. 669-678, Oct. 1972.

[16] J. P. Webb, "Finite element analysis of dispersion in waveguides with sharp metal edges," IEEE Trans. Microwave Theory Tech., vol. 36, pp. 1819-1824, Dec. 1988.

[17] C.-K. Tzuang and T. Itoh, "Finite element analysis of slow-wave Schottky contact printed lines," IEEE Trans. Microwave Theory Tech., vol. MTT-34, pp. 1483-1489, Dec. 1986.

[18] W. Schroeder and I. Wolff, "Full wave boundary integral analysis of arbitrary integrated transmission lines: origin and avoidance of spurious solutions," in Proc. 20th European Microwave Conf. (Budapest), 1990, pp. 829-834.

[19] S. Kosslowski, F. Bögelsack, and I. Wolff, "The application of the point matching method to the analysis of microstrip lines with finite metallization thickness," IEEE Trans. Microwave Theory Tech., vol. 36, pp. 1265-1271, Aug. 1988.

[20] M. Thorburn, A. Agoston, and V. K. Tripathi, "Computation of frequency-dependent propagation characteristics of microstriplike propagation structures with discontinuous layers," IEEE Trans. Microwave Theory Tech., vol. 38, pp. 148-153, Feb. 1990.

[21] D. M. Sheen, S. M. Ali, M. D. Abouzahra, and J. A. Kong, "Application of the three-dimensional finite-difference timedomain method to the analysis of planar microstrip circuits," IEEE Trans. Microwave Theory Tech., vol. 38, pp. 849-857, July 1990.

[22] S. Akhtarzad and P. B. Johns, "Three-dimensional transmission-line matrix computer analysis of microstrip resonators," IEEE Trans. Microwave Theory Tech., vol. MTT-23, pp. 990-997, Dec. 1975.

[23] R. F. Harrington, Time-Harmonic Electromagnetic Fields. New York: McGraw-Hill, 1961, ch. 3, sect. 12, pp. 129-132. 
[24] G. Kowalski and R. Pregla, "Dispersion characteristics of shielded microstrips with finite thickness," Arch. Elek. Übertragung., vol. 25, pp. 193-196, Apr. 1971.

[25] R. Vahldieck and J. Bornemann, "A modified mode-matching technique and its application to a class of quasi-planar transmission lines," IEEE Trans. Microwave Theory Tech., vol. MTT-33, pp. 916-926, Oct. 1985

[26] F. Alessandri, U. Goebel, F. Melai, and R. Sorrentino, "Theoretical and experimental characterization of nonsymmetrically shielded planar waveguides for millimeter-wave circuits," IEEE Trans. Microwave Theory Tech., vol. 37, pp. 2020-2027, Dec. 1989.

[27] C.-K. C. Tzuang, C.-D. Chen, and S. T. Peng, "Full-wave analysis of lossy quasi-planar transmission line incorporating the metal modes," IEEE Trans. Microwave Theory Tech., vol. 38, pp. 1792-1799, Dec. 1990.

[28] Y. Fukuoka, Y.-C. Shih, and T. Itoh, "Analysis of slow-wave coplanar waveguide for monolithic integrated circuits," IEEE Trans Microwave Theory Tech., vol. MTT-31, pp. 567-573, July 1983.

[29] S. W. Lee, W. R. Jones, and J. J. Campbell, "Convergence of numerical solutions of iris-type discontinuity problems," IEEE Trans. Microwace Theory Tech., vol. MTT-19, pp. 528-536, June 1971.

[30] T. S. Chu, T. Itoh, and Y.-C. Shih, "Comparative study of modematching formulations for microstrip discontinuity problems," IEEE Trans. Microwave Theory Tech., vol. MTT-33, pp. 1018-1023, Oct. 1985.

[31] E. Yamashita, and K. Atsuki, "Analysis of microstrip-like transmission lines by nonuniform discretization of integral equations," IEEE Trans. Microwace Theory Tech., vol. MTT-24, pp. 195-200, Apr. 1976.

[32] E. Yamashita, H. Ohashi, and K. Atsuki, "Characterization of microstrip lines near a substrate edge and design formulas of edge-compensated microstrip lines," IEEE Trans. Microwave Theory Tech., vol. 37, pp. 890-896, May 1989.

Ching-Kuang C. Tzuang (S'84-M'86) was born in Taiwan on May 10, 1955. He received the B.S. degree in electronic engineering from the National Chiao Tung University, Hsinchu, Taiwan, in 1977 and the M.S degree from the University of California at Los Angeles in 1980.

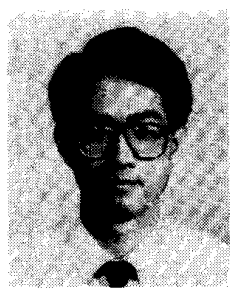

From February 1981 to June 1984, he was with TRW, Redondo Beach, CA, working on analog and digital monolithic microwave integrated circuits. He received the Ph.D. degree in electrical engineering in 1986 from the University of Texas at Austin, where he worked on high-speed transient analyses of monolithic microwave integrated circuits. Since September 1986, he has been with the Institute of Communication Engineering, National Chiao Tung University, Hsinchu, Taiwan, R.O.C. His research activities involve the design and development of millimeter-wave and microwave active and passive circuits and the field theory analysis and design of various quasi-planar integrated circuits.

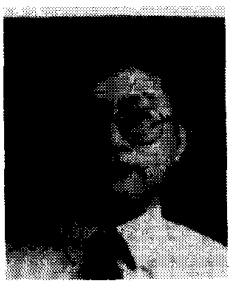

Jan-Dong Tseng ( ' $^{\prime} 9$ ) was born in Taiwan on July 24,1958 . He received the B.S. and M.S. degrees in communication engineering from the National Chiao Tung University, Hsinchu, Taiwan, in 1980 and 1982, respectively.

From 1984 to 1985 , he was with Microelectronics Technology Inc. as a design engineer. From 1985 to 1988 , he was with the Chung Shan Institute of Science and Technology, Lun Tan, Taiwan, as an assistant researcher. His work involved in the design and development of passive and active microwave components. Since 1988 , he has been pursuing the Ph.D. degree at the National Chiao Tung University, Hsinchu, Taiwan. His current research interests include the rigorous field analysis of quasi-planar transmission lines and the design of millimeter-wave components. 